\title{
Effets de six composts sur les réponses physiologiques, biochimiques et agronomiques du niébé Vigna unguiculata L. Walp var. KVX. 61.1. au déficit hydrique
}

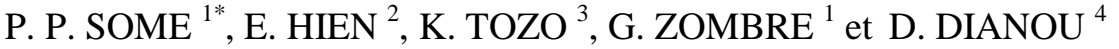 \\ ${ }^{1}$ Laboratoire de Physiologie Végétale, Université de Ouagadougou, UFR/SVT, \\ 03 BP7021, Ouagadougou 03, Burkina Faso. \\ ${ }^{2}$ Institut de Recherche pour le Développement, UMR ECO\&SOLS, BP182 Ouagadougou 01, Burkina Faso. \\ ${ }^{3}$ Université de Lomé Togo, Département de Botanique, Lomé, Togo. \\ ${ }^{4}$ Centre National de Recherche Scientifiques et Technologiques, 03 BP 7047, Ouagadougou, Burkina Faso. \\ *Auteur correspondant ; E-mail : sompepin@yahoo.fr
}

\section{RESUME}

Un déficit hydrique sévère a été imposé par suspension de l'arrosage à deux stades de développement à une variété de niébé Vigna unguiculata L. Walp var. KVX. 61.1 cultivée en pot à l'air libre. Le sol de culture est un Lixisol Ferrique amendé par six types de composts fabriqués à partir de mélanges de déchets urbains solides composés de déchets verts (DV), de déchets d'abattoir (DA), de déchets de cuisine (DC) selon les compositions $\quad$ suivantes: $\quad \mathrm{C} 1=40 \% \mathrm{DV}+60 \% \mathrm{DA} ; \quad \mathrm{C} 2=40 \% \mathrm{DV}+40 \% \mathrm{DA}+20 \% \mathrm{DC}$; $\mathrm{C} 3=40 \% \mathrm{DV}+30 \% \mathrm{DA}+30 \% \mathrm{DC} ; \mathrm{C} 4=40 \% \mathrm{DV}+20 \% \mathrm{DA}+40 \% \mathrm{DC} ; \mathrm{C} 5=40 \% \mathrm{DV}+60 \% \mathrm{DC} ; \mathrm{C} 6=100 \% \mathrm{DV}$. Les effets de ces six composts sur le potentiel hydrique, la fuite d'électrolyte, la teneur en amidon des feuilles et sur quelques paramètres agronomiques ont été évalués sur les plantes stressées en comparaison avec les plantes témoins. Les résultats indiquent des valeurs du potentiel hydrique foliaire très faibles et une réduction de la teneur en amidon des feuilles pour les plantes des composts $\mathrm{C} 2, \mathrm{C} 3, \mathrm{C} 4$, et $\mathrm{C} 5$. Ces dernières présentent des intégrités membranaires plus affectées comparativement aux plantes du sol témoin et celles des composts $\mathrm{C} 1$ et C6. Les composts C2, C3, C4 et C5 favorisent le développement des racines et augmentent le rendement en graines du niébé en condition de déficit hydrique. Ce qui justifie l'intérêt du compostage des déchets de cuisine.

(C) 2014 International Formulae Group. All rights reserved.

Mots clés : niébé, déficit hydrique, compost, déchets urbains solides.

\section{INTRODUCTION}

Le niébé (Vigna unguiculata (L.) Walp.) est une importante légumineuse à graines et une source majeure de minéraux et de protéines alimentaires pour les populations urbaines et rurales et leur bétail dans les tropiques d'Afrique, d'Asie, et d'Amérique (Singh et Awika, 2010). La région du Sahel fournit $64 \%$ de la production mondiale de niébé estimée à 12,5 millions de tonnes sur une superficie totale de 14 millions d'hectares (Singh et al., 2003; Agbicodo et al., 2009). Le niébé est relativement productif dans les environnements marginaux caractérisés par des sols dégradés et des pluviométries faibles et erratiques (400-600 mm/an) (Ogbonnaya et 
al., 2003). Il contribue à la restauration de la fertilité des terres par sa capacité à fixer l'azote atmosphérique, et constitue ainsi un élément essentiel dans les stratégies de gestion durable des terres dans les systèmes agricoles pluviaux (Fatokun et al., 2009). Malgré sa réputation de plante résistante à la sécheresse, les déficits hydriques récurrents réduisent significativement sa croissance et sa production (Hall et al., 2003; Muchero et al., 2008). Les modèles de climat prédisent que les changements climatiques attribués aux émissions des gaz à effet de serre dues à la déforestation, à l'industrialisation et à l'urbanisation vont augmenter la fréquence et l'intensité des sécheresses (Wittig et al., 2007; Collins et al., 2008; Salack et al., 2011), avec des conséquences plus dramatiques sur l'agriculture et la sécurité alimentaire dans les pays en développement ( IPCC, 2007; Yang et al., 2010). Au Burkina Faso, les zones sahélienne et soudanienne sont les plus affectées par la sécheresse. Drabo et al. (1993) montrent que la zone sahélienne est caractérisée par des poches de sécheresse de dix à plus de quinze jours, des températures élevées $\left(38^{\circ} \mathrm{C}\right.$ à $\left.45^{\circ} \mathrm{C}\right)$ tant de l'air que du sol et que celle de la savane soudanienne est caractérisée par des poches de sécheresse de cinq à dix jours.

A ces contraintes climatiques vient s'ajouter l'appauvrissement des sols cultivés en matière organique et en éléments minéraux. Kaboré et al. (2010) montrent que la poussée démographique a engendré une pression humaine jamais égalée sur les ressources naturelles renouvelables. Les terres cultivables et même les terres marginales dans les zones de forte densité sont mises en culture en permanence. La mise en culture continue, conjuguée avec les contraintes climatiques entraîne un appauvrissement des sols en matière organique et en éléments minéraux et un effondrement de la structure (Hien, 2004), la perte de la productivité et l'abandon des terres par les paysans. Dans le plateau central à plus forte pression humaine, la dégradation des terres est plus accentuée qu'ailleurs.

Les récentes préoccupations sur les effets des engrais chimiques sur l'environnement, leurs prix élevés ont rendu urgent le développement d'une approche rationnelle vers le choix des engrais organiques.

Au Burkina Faso, il existe un potentiel non négligeable de pratiques de fumure et d'amendement des sols à même de favoriser le stockage du carbone dans les sols (Hien, 2004). Toutefois, les pratiques les plus courantes sont celles qui combinent la lutte contre l'érosion et la concentration de la fumure organique ou minérale. Depuis quelques années, plusieurs travaux ont décrit l'utilisation de substrats organiques (SO), particulièrement les déchets urbains solides (DUS) pour l'agriculture dans la ville de Ouagadougou et sa périphérie par des acteurs divers que sont les céréaliers, les maraîchers, les pépiniéristes (Kabore, 2004). Dans un contexte de forte dégradation des sols et de coût élevé des engrais chimiques, le recours aux SO en général dans l'agriculture périurbaine s'est présenté comme une alternative intéressante pour la fertilisation des sols cultivés.

Les composts sont en effet des produits riches en matières organiques et également en composés minéraux $(\mathrm{N}, \mathrm{P}, \mathrm{K}, \mathrm{Mn}$, oligoéléments,...) et à ce titre sont susceptibles d'améliorer la fertilité du sol (Soumaré et al., 2003). Cependant, il est souvent difficile de voir si cette amélioration de la fertilité du sol est due à la présence d'éléments minéraux fertilisants au sein du compost, à leur minéralisation progressive, à l'amélioration des caractéristiques physiques du sol ou à leur capacité d'augmenter le potentiel hydrique du sol. Ce travail a été initié afin de vérifier l'effet de différents composts sur le potentiel hydrique du sol à travers une étude des réponses physiologiques, biochimiques et agronomiques du niébé (Vigna unguiculata L. 
Walp. var. KVX61.1) soumis au stress hydrique.

\section{MATERIEL ET METHODES Matériel}

Le matériel végétal utilisé est la variété de niébé Vigna unguiculata L. Walp var. KVX61-1 qui a un cycle court de 70 jours. Le sol témoin utilisé dans l'essai est un Lixisol Ferrique ((FAO-ISRIC-ISSS, 2006) du site expérimental de Gampéla (25 km à l'Est de Ouagadougou, $\left.1^{\circ} 21^{\prime} \mathrm{O} ; 12^{\circ} 24^{\prime} \mathrm{N}\right)$. Ce sol est représentatif des sols de la zone périurbaine dont les caractéristiques physiques et chimiques sont présentées dans le Tableau 1. Le prélèvement a été effectué dans la couche $0-10 \mathrm{~cm}$.

Les six composts utilisés dans l'expérimentation ont été produits par l'Unité Mixte de Recherche (UMR) Ecologie Fonctionnelle \& Biogéochimie des sols \& des Agro-écosystèmes de l'IRD à Ouagadougou en 2009 à base de déchets urbains solides (DUS) de la ville de Ouagadougou. Ces déchets sont composés essentiellement de déchets verts (DV) constitués de feuilles de Kaya senegalensis, de déchets d'abattoir (DA) et de déchets de cuisine (DC) constitués de légumes. Les pourcentages de ces DUS dans chaque type de compost figurent dans le Tableau 2. Dans les mélanges de substrats organiques, la proportion de déchets verts (DV) est maintenue constante parce que c'est le substrat le plus lignifié, donc le plus difficilement minéralisable. Les proportions de DA et DC sont variables parce qu'il est bien établi que dans un compostage, l'augmentation de la proportion d'éléments organiques fortement humifiés ou fortement hydratés dans les substrats compostés, permet d'accélérer la minéralisation de déchets organiques riches en lignine et cellulose, et détermine la qualité chimique et biochimique $\mathrm{du}$ compost final. Les composts finaux contenant plus de DA et/ou de DC (1,2 et 3$)$ sont plus humifiés et minéralisés par rapport aux autres (4, 5 et 6$)$. Le compost 6 , produit exclusivement à partir de DV ne contient pas de nitrates.

Le compostage a duré seize semaines à l'issue desquelles les composts finaux ont présenté les caractéristiques physiques et chimiques données dans le Tableau 3.

\section{Température et humidité relative}

Pour évaluer l'impact des contraintes environnementales, la température et l'humidité relative ont été enregistrées quotidiennement à $8 \mathrm{~h}, 12 \mathrm{~h}$ et $17 \mathrm{~h}$ durant l'application du stress au stade végétatif et au stade de floraison. La moyenne journalière de ces trois valeurs montre que les températures varient entre $30^{\circ}$ et $35^{\circ} \mathrm{C}$, et l'humidité comprise entre $45 \%$ et $55 \%$ pendant la période d'application du stress au stade végétatif, alors qu'au stade floraison les températures sont de $25^{\circ}$ à $34^{\circ}$ et l'humidité $60 \%$ à $85 \%$.

\section{Méthodes \\ Dispositif expérimental}

L'essai a été réalisé selon un dispositif en split splot à 4 répétitions avec deux facteurs que sont l'amendement et le régime hydrique. Le facteur amendement est défini par $\mathrm{C}_{0}$, sol témoin sans compost et $\mathrm{C}_{1}, \mathrm{C}_{2}, \mathrm{C}_{3}$, $\mathrm{C}_{4}, \mathrm{C}_{5}, \mathrm{C}_{6}$, sols amendés avec respectivement les composts 1, 2, 3, 4, 5, 6 (Tableau 3). Le régime hydrique est à trois niveaux qui sont $\mathrm{T} 0=$ témoin avec une alimentation hydrique non limitante, $\mathrm{T} 1=$ suspension d'irrigation durant la phase végétative et $\mathrm{T} 2=$ suspension d'irrigation en phase de floraison. L'unité expérimentale est constituée de 3 pots.

\section{Conduite de l'étude}

Les semis ont été effectués le 20 Juin 2010 dans des pots de 6 litres en plastique à fond troué, préalablement remplis de $6 \mathrm{~kg}$ de terre provenant du sol dégradé (sol de culture). Cette terre a été séchée à l'air et tamisée à $2 \mathrm{~mm}$. Elle a été ensuite mélangée à $150 \mathrm{~g}$ de chacun des six (6) types de compost et arrosée la veille à la capacité au champ (CAC). Quatre (4) graines par pot ont été semées et les plantules ont été démariées à une plante par pot 14 jours après semis (JAS).

Trois régimes hydriques (T0, T1, T2) ont été appliqués: un tiers (1/3) des pots correspondant aux témoins est arrosé quotidiennement à la capacité au champ (500 ml d'eau) jusqu'à la fin de l'essai (T0). Au $26^{\text {è }}$ JAS (phase végétative), le stress hydrique a 
été appliqué au second tiers des pots (T1) et à $50 \%$ de floraison le tiers restant des pots, a subi le stress hydrique (T2).

Le stress hydrique appliqué a consisté à suspendre l'arrosage des pots des traitements concernés et à les recouvrir en cas de pluie d'un hangar mobile dont le toit est fait en tôle. Le stress T1 a été appliqué à tous les types d'amendement du $26^{\mathrm{e}}$ au $42^{\mathrm{è}} \mathrm{JAS}$ au stade végétatif (15 au 31 Juillet). T2 a été appliqué à tous les types d'amendement du $52^{\mathrm{e}}$ au $65^{\mathrm{e}} \mathrm{JAS}$ au stade floraison (du 9 au 22 Août). La veille du début du stress hydrique, les plantes témoins et celles à stresser ont été arrosées à la capacité au champ. A la fin des stress $\mathrm{T} 1$ et $\mathrm{T} 2$, les plantes ont été réarrosées jusqu'à la fin de l'essai.

\section{Paramètres mesurés Le potentiel hydrique foliaire}

Le potentiel hydrique foliaire est mesuré par la technique de la chambre à pression de Scholander (modèle SKPM 1400, Skye Instruments, Powys, UK) sur la première jeune feuille, entièrement développée. La feuille, fraîchement récoltée, est insérée dans un bouchon pour en assurer son étanchéité et immédiatement placée dans la chambre. L'extrémité coupée dépasse de quelques millimètres à l'extérieur du bouchon afin de pouvoir observer l'affleurement de la sève au niveau de la section des vaisseaux. La pression appliquée est augmentée doucement dans la chambre jusqu'à ce que la sève apparaisse. La pression qui provoque la sortie de la première goutte, exprimée en $\mathrm{MPa}$ correspond à l'opposé du potentiel hydrique de la feuille. Pour plus de précision, l'observation se fait à la loupe binoculaire.

\section{La perméabilité membranaire}

La perméabilité membranaire a été mesurée par le test de fuite d'électrolytes. Ce dernier est basé sur la mesure de l'électroconductivité d'un milieu aqueux où des échantillons de feuilles stressées et non stressées ont séjourné.

L'efflux d'électrolytes a été mesuré selon le protocole de Thiaw (2003). Ainsi, cinq disques foliaires sont prélevés à l'emporte-pièce sur la troisième feuille (à partir de l'apex) d'une plante de chaque unité expérimentale. Ces disques sont mis dans des boîtes de Pétri pour être rincés à l'eau distillée avant d'être placés dans des tubes à essai contenant $10 \mathrm{ml}$ d'eau distillée. L'ensemble est immergé dans un bain marie à $45{ }^{\circ} \mathrm{C}$ pendant 2 heures. La conductivité $(\mathrm{C} 1)$ de la solution est alors mesurée avec un conductimètre préalablement étalonné. L'échantillon est ensuite mis au bain marie bouillant $\left(100{ }^{\circ} \mathrm{C}\right)$ pendant $20 \mathrm{mn}$, puis refroidi à la température ambiante. La conductivité (C2) est mesurée une seconde fois. Le pourcentage de fuite d'électrolytes (FE \%) est calculé suivant la formule (Tripathy et al., 2000): FE (\%) = (C1/C2)x100

\section{Détermination de la teneur en amidon}

La teneur en amidon a été déterminée selon la méthode de Jarnis et Walker (1993) dans les deux folioles latérales des 5è feuilles séchées et broyées en poudre.

\section{Les paramètres agronomiques}

Pour obtenir le rapport partie racinaire sur partie aérienne (PR/PA), les biomasses sèches racinaire et aérienne de chaque plant et pour chaque traitement ont été déterminées après séchage des racines, tiges et feuilles à l'étuve à $80{ }^{\circ} \mathrm{C}$ pendant $48 \mathrm{~h}$. La partie racinaire a été préalablement débarrassée des particules de sol par lavage dans un tamis à mailles fines.

La récolte des gousses a été progressive et a été effectuée sur toutes les plantes. Les gousses récoltées ont été séchées au laboratoire sur une paillasse. Après séchage, le nombre de gousses par plante $\left(\right.$ ngse $\left.\mathrm{pt}^{-1}\right)$, le poids sec des gousses par plante (pgse $\mathrm{pt}^{-1}$ ) ont été déterminés. Les gousses ont été ensuite décortiquées pour déterminer le nombre de graines total par plante $\left(\mathrm{ngr} \mathrm{pt}^{-1}\right)$ et le poids sec des graines par plante $\left(\mathrm{pgr}^{\mathrm{pt}} \mathrm{pt}^{-1}\right.$.

\section{Analyse des résultats}

Les calculs de moyenne des quatre répétitions et les graphiques ont été réalisés sur un tableur Excel 2007. Les analyses statistiques des variables mesurées ont été faites avec le logiciel XLSTAT-Pro version 
7.5. Les données ont été soumises à l'analyse de la variance (ANOVA) à deux facteurs (amendement et régime hydrique) et les moyennes ont été comparées par le test de Student Newman-Keuls au seuil de 5\%. Le test de signification des coefficients de corrélation de Person a été effectué entre la teneur en amidon des feuilles, le pourcentage de fuite d'électrolytes et le potentiel hydrique foliaire toujours avec logiciel XLSTAT-Pro.

\section{RESULTATS}

Effet du stress hydrique sur le potentiel hydrique foliaire

La Figure 1 montre que le potentiel hydrique est plus faible chez les plantes stressées que chez les témoins aux stades végétatifs et floraison. Les plantes stressées du sol témoin $\mathrm{C} 0$ et des composts $\mathrm{C} 3$ et $\mathrm{C} 6$ ont les valeurs les plus élevées avec en moyenne 0,6 MPa en T1 et -0,9MPa en T2. Les plus faibles valeurs sont observées chez les plantes des composts $\mathrm{C} 1, \mathrm{C} 2, \mathrm{C} 4$ et $\mathrm{C} 5$ avec une moyenne de -1,4MPa en $\mathrm{T} 1$ et $-1,6 \mathrm{MPa}$ en $\mathrm{T} 2$. Ces deux groupes diffèrent significativement en situation de stress et non stress. L'analyse statistique (Tableau 4 ) révèle que le régime hydrique ( $\mathrm{RH})$ et le type de compost ont des effets significatifs sur le potentiel hydrique

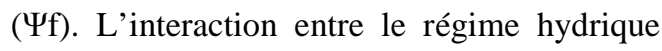
$(\mathrm{Rh})$ et le type de compost n'a eu un effet significatif sur le $\Psi$ f qu'au stade de floraison.

\section{Effet du stress hydrique sur l'intégrité membranaire}

Les résultats de la Figure 2 montrent que le pourcentage de fuite d'électrolytes (FE\%) est plus élevé chez les plantes stressées que chez les plantes témoins en phase végétative $(\mathrm{A})$ et en phase de floraison (B) sauf pour le compost C6 (en B).

En considérant le facteur compost, l'analyse de la variance des fuites d'électrolytes n'a révélé aucune différence significative chez les plantes en conditions témoins tandis qu'en conditions de stress hydrique (T1 et T2), les composts $\mathrm{C} 0, \mathrm{C} 1 \mathrm{et}$
C6 diffèrent significativement de C2, C3, C4 et C5. L'analyse de la variance (Tableau 4) montre que le régime hydrique, le type de compost et leur interaction ont eu un effet significatif $\quad(p<0.0001) \quad$ sur $\quad$ l'intégrité membranaire.

\section{Effet du stress hydrique sur la teneur en amidon des feuilles}

La teneur en amidon des feuilles des plants stressés à tous les niveaux d'amendement a été affectée par le déficit hydrique à T1 et à T2 (Figure 3). L'effet du manque d'eau s'est traduit par une diminution de la teneur en amidon des feuilles des plantes stressées par rapport à celles non stressées aux deux stades de développement. Cette diminution a été plus importante à T2 qu'à T1. En effet, le taux de réduction de la teneur en amidon des feuilles à T1 a été de 35,80\% pour les plantes du sol témoin $\mathrm{C} 0$ alors qu'à $\mathrm{T} 2$ elle a été de $51,97 \%$ pour les plantes du compost C6. Les composts C4 et C5 ont plus favorisé une accumulation de l'amidon dans les feuilles avec respectivement une augmentation de $73,17 \%$ et $80,11 \%$ à $\mathrm{T} 1$ et $81,59 \%$ et $98,84 \%$ à $\mathrm{T} 2$ des plantes stressées par rapport au sol témoin.

L'analyse de la variance (Tableau 4) montre que le régime hydrique, le type de compost et leur interaction ont eu un effet significatif $(\mathrm{p}<0.0001)$ sur la teneur en amidon des feuilles.

\section{Relations en les paramètres physiologiques et biochimiques}

Le Tableau 5 présente les coefficients de corrélation entre la teneur en amidon des feuilles, le pourcentage de fuite d'électrolytes et le potentiel hydrique foliaire. Au stade floraison, la teneur en amidon est corrélée négativement $(r=-0,83)$ avec le potentiel hydrique chez les plantes témoins et positivement $(r=0,89)$ chez les stressées. Par contre, la corrélation n'est pas significative entre la teneur en amidon et le pourcentage de 
fuite d'électrolytes à tous les stades. Le potentiel hydrique foliaire est corrélé négativement au pourcentage de fuite d'électrolytes chez les plantes stressées au stade végétatif $(r=-0,73)$ et floraison $(r=-$ $0.81)$.

Effet du stress hydrique sur les paramètres agronomiques

\section{Rapport partie racinaire sur partie aérienne}

La Figure 4A illustre l'effet de l'intensité du stress hydrique et de sa durée sur le rapport partie racinaire sur partie aérienne (PR/PA). Ce rapport est plus élevé chez les plantes stressées que chez les plantes non stressées (Figure 6B). L'augmentation du rapport PR/PA s'est accentuée au cours de l'application du stress hydrique au stade floraison chez les plantes stressées. Par contre, en comparaison avec les plantes du sol témoin, ce rapport a connu une baisse au niveau des composts $\mathrm{C} 1, \mathrm{C} 2$, et $\mathrm{C} 4$. L'effet régime hydrique $(\mathrm{RH})$ est significatif $(\mathrm{p}<0,0001)$ sur le rapport PR/PA au stade floraison et non significatif au stade végétatif. Le type de compost et l'interaction RHxCompost ont eu un effet significatif sur ce rapport à tous les stades de développement (Tableau 6).

\section{Composantes du rendement}

Les variables agronomiques étudiées après la récolte ont été significativement réduites par le déficit hydrique chez les plantes des composts $\mathrm{C} 1, \mathrm{C} 2, \mathrm{C} 4$ et $\mathrm{C} 5$. Celles du sol témoin $\mathrm{C} 0$ et des composts C3, C6 n'ont pas subi une réduction significative. L'analyse statistique des résultats des composantes du rendement étudiées révèlent une différence significative $(\mathrm{P}<0,0001)$ entre le sol témoin $\mathrm{C} 0$, le compost $\mathrm{C} 6$ et les autres, quel que soit le régime hydrique, sauf le nombre de gousses des plantes T2. L'examen du Tableau 7 montre que les plantes du sol témoin $\mathrm{C} 0$ et du compost $\mathrm{C} 6$ possèdent les valeurs des variables agronomiques les plus faibles, tandis que celles de C1, C2, C4 et C5 possèdent les valeurs les plus élevées.

Les effets régime hydrique $(\mathrm{RH})$ au stade végétatif et floraison, compost et l'interaction RHxCompost sont significatifs $(\mathrm{p}<0,0001)$ sur le nombre de gousses, le poids sec des gousses, le nombre de graines et le poids sec des graines (Tableau 8).

Tableau 1: Caractéristiques physico-chimiques initiales de l'horizon superficiel $(0-10 \mathrm{~cm}) \mathrm{du}$ sol de culture.

\begin{tabular}{lccccccc}
\hline Sables & Limons & Argiles & $\begin{array}{c}\text { Carbone } \\
\text { organique }\end{array}$ & $\begin{array}{c}\text { Azote } \\
\text { total }\end{array}$ & pH eau & $\begin{array}{c}\text { Somme des bases } \\
\text { échangeables }\end{array}$ & CEC \\
\hline $68,8 \%$ & $20,3 \%$ & $10,9 \%$ & $3,0 \mathrm{~g} \cdot \mathrm{kg}^{-1}$ & $0,2 \mathrm{~g} \cdot \mathrm{kg}^{-1}$ & 5,3 & $1,52 \mathrm{cmol} \cdot \mathrm{kg}^{-1}$ & $2 \mathrm{cmol}^{-1} \mathrm{~kg}^{-1}$ \\
\hline
\end{tabular}

Tableau 2 : Nature et pourcentage des substrats initiaux utilisés dans chaque type de compost.

\begin{tabular}{lcccccc}
\hline DUS (en \%) & \multicolumn{7}{c}{ Compost } \\
\cline { 2 - 7 } & $\mathbf{C}_{\mathbf{1}}$ & $\mathbf{C}_{\mathbf{2}}$ & $\mathbf{C}_{\mathbf{3}}$ & $\mathbf{C}_{\mathbf{4}}$ & $\mathbf{C}_{\mathbf{5}}$ & $\mathbf{C}_{\mathbf{6}}$ \\
\hline DV & 40 & 40 & 40 & 40 & 40 & 100 \\
DA & 60 & 40 & 30 & 20 & 0 & 0 \\
DC & 0 & 20 & 30 & 40 & 60 & 0 \\
\hline
\end{tabular}

DUS : déchets urbains solides; DV : déchets verts ; DA : déchets d'abattoir ; DC : déchets de cuisine. 
Tableau 3 : Caractéristiques physiques et chimiques des six composts.

\begin{tabular}{|c|c|c|c|c|c|c|c|}
\hline \multirow[t]{2}{*}{ Compost } & \multicolumn{3}{|c|}{ g.kg ${ }^{-1}$ de MS } & \multicolumn{2}{|c|}{ mg.kg ${ }^{-1}$ de MS } & \multirow[t]{2}{*}{$\mathrm{C} / \mathrm{N}$} & \multirow[t]{2}{*}{ pH } \\
\hline & MOT & C & $\mathbf{N}$ & $\mathrm{N}-\mathrm{NH}_{4}{ }^{+}$ & $\mathrm{N}-\mathrm{NO}_{3}^{-}$ & & \\
\hline $\mathrm{C} 1$ & 550 & 265,7 & 13,9 & 8,8 & 1,4 & 19,1 & 8,9 \\
\hline $\mathrm{C} 2$ & 390 & 270,1 & 14,8 & 8,9 & 1,7 & 18,2 & 8,7 \\
\hline C3 & 576 & 302,2 & 14,7 & 8,4 & 2,1 & 20,6 & 8,5 \\
\hline $\mathrm{C} 4$ & 363 & 280,2 & 15,4 & 8,1 & 7,0 & 18,2 & 8,7 \\
\hline C5 & 462 & 380,3 & 20,7 & 11,0 & 1,2 & 18,4 & 8,4 \\
\hline C6 & 731 & 425,5 & 16,5 & 6,0 & 0,0 & 25,8 & 8,2 \\
\hline $\begin{array}{l}\text { Source: } \\
\text { MOT : } \\
\text { nitrique } \\
\mathrm{C}_{1}=60 \% \\
\mathrm{C}_{4}=20 \% \\
\mathrm{DV}: \text { dé } \\
\text { légumes }\end{array}$ & $\begin{array}{l}\text { iité Mixte } \\
\text { ière organi } \\
/ \mathrm{N}: \text { Rappo } \\
\mathrm{V}+40 \% \mathrm{D} \\
\mathrm{V}+40 \% \mathrm{D} \\
\text { ts verts cor }\end{array}$ & $\begin{array}{l}\text { herche Ec } \\
\text { tale; } \mathrm{C}: \mathrm{Ci} \\
\text { one/azote } \\
=40 \% \mathrm{DV} \\
\% \mathrm{DC} ; \mathrm{C}_{5}= \\
\text { de feuille }\end{array}$ & $\begin{array}{l}\text { biogéo } \\
\text { etotal } \\
\text { : potent } \\
\% \text { DA + } \\
\text { oV + 40 } \\
\text { rbres ; D }\end{array}$ & $\begin{array}{l}\text { Imie des Sols } \\
\text { Azote total } \\
\text { en ions hydro } \\
\% \text { DC; } \mathrm{C}_{3}=4 \\
\mathrm{DA}+60 \% \mathrm{DC} \\
: \text { déchets d'a }\end{array}$ & $\begin{array}{l}\text { MR Eco \& } \\
\mathrm{NH}_{4}^{+}: \text {Azot } \\
\text { le ; } \\
\text { PV }+30 \% \mathrm{D} \\
6=100 \% \mathrm{DV} \\
\text { oir ; DC : d }\end{array}$ & $\begin{array}{l}\text { e l'IRD } \\
\text { oniacal } \\
\text { Matière } \\
0 \% \text { DC; } \\
\text { de cuis }\end{array}$ & $\begin{array}{l}\text { Faso) } \\
3^{-} \text {: Azote } \\
\text { tués de }\end{array}$ \\
\hline
\end{tabular}

Tableau 4 : Analyse de la variance du potentiel hydrique foliaire ( $\Psi f$ ), du pourcentage de fuites d'électrolytes (FE\%) et de la teneur en amidon au stade végétatif et au stade floraison.

\begin{tabular}{|c|c|c|c|c|c|c|c|c|}
\hline \multirow{3}{*}{ Source } & \multirow{3}{*}{ ddl } & \multicolumn{4}{|c|}{ Stade végétatif } & \multicolumn{3}{|c|}{ Stade floraison } \\
\hline & & $\Psi \mathbf{f}$ & FE\% & \multicolumn{2}{|c|}{ Amidon } & $\Psi f$ & FE\% & Amidon \\
\hline & & CM F & CM F & $\mathbf{C M}$ & $\mathbf{F}$ & $\mathbf{C M}$ & $\mathbf{C M}$ & $\mathbf{C M}$ \\
\hline $\mathrm{Rh}$ & 1 & $\begin{array}{l}0,15 \quad * * \\
\mathrm{p}=0,006\end{array}$ & $\begin{array}{l}1281,22 * * \\
\mathrm{p}<0,0001\end{array}$ & $\begin{array}{l}7758 \\
\mathrm{p}<0,\end{array}$ & $\begin{array}{l}4 * * \\
01\end{array}$ & $\begin{array}{l}4,42 \quad * * \\
\mathrm{p}<0,0001\end{array}$ & $\begin{array}{l}4908,02 * * \\
\mathrm{p}<0,0001\end{array}$ & $\begin{array}{l}4866,34 \\
\mathrm{p}<0,0001\end{array}$ \\
\hline Compost & 6 & $\begin{array}{l}1,19 \quad * * \\
\mathrm{p}<0,0001\end{array}$ & $\begin{array}{l}301,08 * * \\
\mathrm{p}<0,0001\end{array}$ & $\begin{array}{l}396, \\
\mathrm{p}<0,\end{array}$ & & $\begin{array}{l}2,33 \quad * * \\
\mathrm{p}<0,0001\end{array}$ & $\begin{array}{l}742,06 * * \\
\mathrm{p}<0,0001\end{array}$ & $\begin{array}{l}189,88 * * * \\
\mathrm{p}<0,0001\end{array}$ \\
\hline Rh*Compost & 6 & $\begin{array}{l}0,01 \mathrm{~ns} \\
\mathrm{p}=0,940\end{array}$ & $\begin{array}{l}25,26 * * \\
\mathrm{p}<0,0001\end{array}$ & $\begin{array}{l}448, \\
\mathrm{p}<0,\end{array}$ & & $\begin{array}{l}0,18 \quad * * \\
\mathrm{p}<0,0001\end{array}$ & $\begin{array}{l}221,83 \\
* * \\
\mathrm{p}<0,0001\end{array}$ & $\begin{array}{l}291,95 * * * \\
\mathrm{p}<0,0001\end{array}$ \\
\hline
\end{tabular}

$\mathrm{CM}=$ carré moyen $; \mathrm{Rh}=$ régime hydrique $; \mathrm{F}=$ facteur de Fisher $; *=$ significatif au seuil de $5 \% ; * *=$ significatif au seuil de $1 \% ; \mathrm{ns}=$ non significatif.

Tableau 5: Corrélation entre la teneur en amidon des feuilles, le pourcentage de fuite d'électrolytes et le potentiel hydrique foliaire.

\begin{tabular}{lccccc}
\hline \multirow{2}{*}{ Paramètres testés } & \multicolumn{2}{c}{ Stade végétatif } & \multicolumn{2}{c}{ Stade floraison } \\
\cline { 3 - 5 } & & Témoins & Stressées & Témoins & Stressées \\
\hline Amid & If & $-0,72$ & 0,42 & $\mathbf{- 0 , 8 3}$ & $\mathbf{0 , 8 9}$ \\
Amid & FE & 0,568 & $-0,277$ & $-0,221$ & $-0,646$ \\
If & FE & $-0,103$ & $\mathbf{- 0 , 7 3 9}$ & 0,099 & $\mathbf{- 0 , 8 1}$ \\
\hline \multicolumn{2}{l}{ Chiffres en gras } & $=$ \\
\end{tabular}


Tableau 6 : Analyse de la variance du rapport partie racinaire sur partie aérienne (PR/PA) au stade végétatif et au stade floraison.

\begin{tabular}{|c|c|c|c|}
\hline \multirow{3}{*}{ Source } & \multirow{3}{*}{ ddl } & Stade végétatif & \multirow{2}{*}{$\begin{array}{c}\begin{array}{c}\text { Stade } \\
\text { floraison }\end{array} \\
\text { PR/PA }\end{array}$} \\
\hline & & PR/PA & \\
\hline & & $\mathbf{C M}$ & CM \\
\hline $\mathrm{Rh}$ & 1 & $\begin{array}{c}0,001 \mathrm{~ns} \\
\mathrm{p}=0,702\end{array}$ & $\begin{array}{c}0,139 \quad * * \\
\mathrm{p}<0,0001\end{array}$ \\
\hline Compost & 6 & $\begin{array}{l}0,012 * * \\
\mathrm{p}<0,0001\end{array}$ & $\begin{array}{c}0,008 \quad * * \\
\mathrm{p}<0,0001\end{array}$ \\
\hline $\mathrm{Rh} *$ Compost & 6 & $\begin{array}{l}0,010 \quad * * \\
\mathrm{p}<0,0001\end{array}$ & $\begin{array}{l}0,002 \quad * * \\
\mathrm{p}<0,0001\end{array}$ \\
\hline
\end{tabular}

Tableau 7 : Moyennes des paramètres de production des plantes en conditions témoin (T0) et de stress aux stades végétatif (T1) et floraison (T2) pour les six composts et le sol témoin.

\begin{tabular}{|c|c|c|c|c|c|}
\hline Compost & RH & ngse pt $^{-1}$ & pgse pt $^{-1}$ & ngrs pt $^{-1}$ & psgrs pt $^{-1}$ \\
\hline \multirow{3}{*}{$\mathrm{C}_{0}$} & T0 & $3^{\mathrm{c}}$ & $1,48^{\mathrm{d}}$ & $10^{\mathrm{c}}$ & $1,15^{\mathrm{c}}$ \\
\hline & $\mathrm{T} 1$ & $1^{\mathrm{c}}$ & $1,17^{\mathrm{c}}$ & $8^{d}$ & $1,02^{\mathrm{d}}$ \\
\hline & $\mathrm{T} 2$ & $2^{a}$ & $1,06^{\mathrm{c}}$ & $7^{\mathrm{b}}$ & $0,58^{\mathrm{c}}$ \\
\hline \multirow{3}{*}{$\mathrm{C}_{1}$} & T0 & $6^{b}$ & $5,01^{b}$ & $25^{b}$ & $3,14^{b}$ \\
\hline & $\mathrm{T} 1$ & $3^{a b}$ & $2,78^{b}$ & $16^{\mathrm{c}}$ & $2,25^{\mathrm{b}}$ \\
\hline & $\mathrm{T} 2$ & $2^{\mathrm{a}}$ & $1,37^{\mathrm{b}}$ & $9^{\mathrm{b}}$ & $0,89^{\mathrm{b}}$ \\
\hline \multirow{3}{*}{$\mathrm{C}_{2}$} & T0 & $8^{a}$ & $4,85^{\mathrm{a}}$ & $35^{\mathrm{a}}$ & $4,81^{\mathrm{a}}$ \\
\hline & $\mathrm{T} 1$ & $4^{a}$ & $4,08^{\mathrm{a}}$ & $26^{\mathrm{a}}$ & $3,79^{\mathrm{a}}$ \\
\hline & $\mathrm{T} 2$ & $2^{\mathrm{a}}$ & $3,04^{\mathrm{a}}$ & $13^{\mathrm{a}}$ & $1,53^{\mathrm{a}}$ \\
\hline \multirow{3}{*}{$\mathrm{C}_{3}$} & T0 & $4^{b}$ & $2,95^{\mathrm{c}}$ & $26^{b}$ & $2,87^{b}$ \\
\hline & $\mathrm{T} 1$ & $3^{a b}$ & $2,78^{\mathrm{b}}$ & $19^{b}$ & $2,35^{\mathrm{b}}$ \\
\hline & $\mathrm{T} 2$ & $2^{\mathrm{a}}$ & $1,89^{\mathrm{ab}}$ & $12^{\mathrm{a}}$ & $1,42^{b}$ \\
\hline \multirow{3}{*}{$\mathrm{C}_{4}$} & T0 & $8^{a}$ & $5,94^{\mathrm{ab}}$ & $38^{\mathrm{a}}$ & $4,54^{\mathrm{a}}$ \\
\hline & $\mathrm{T} 1$ & $4^{a}$ & $3,12^{\mathrm{a}}$ & $21^{\mathrm{a}}$ & $2,55^{\mathrm{ab}}$ \\
\hline & $\mathrm{T} 2$ & $2^{\mathrm{a}}$ & $2,19^{\mathrm{ab}}$ & $18^{\mathrm{a}}$ & $2,64^{\mathrm{b}}$ \\
\hline \multirow{3}{*}{$\mathrm{C}_{5}$} & T0 & $8^{a}$ & $5,55^{\mathrm{ab}}$ & $36^{\mathrm{a}}$ & $3,32^{\mathrm{a}}$ \\
\hline & $\mathrm{T} 1$ & $6^{\mathrm{a}}$ & $3,23^{\mathrm{a}}$ & $20^{\mathrm{a}}$ & $2,27^{\mathrm{ab}}$ \\
\hline & $\mathrm{T} 2$ & $3^{\mathrm{a}}$ & $1,89^{\mathrm{ab}}$ & $17^{\mathrm{a}}$ & $1,95^{\mathrm{b}}$ \\
\hline \multirow{3}{*}{$\mathrm{C}_{6}$} & T0 & $2^{\mathrm{c}}$ & $1,2^{\mathrm{d}}$ & $11^{\mathrm{c}}$ & $1,28^{\mathrm{c}}$ \\
\hline & $\mathrm{T} 1$ & $2^{\mathrm{c}}$ & $1,13^{\mathrm{c}}$ & $8^{\mathrm{d}}$ & $0,89^{d}$ \\
\hline & $\mathrm{T} 2$ & $2^{\mathrm{a}}$ & $1,47^{\mathrm{c}}$ & $7^{\mathrm{b}}$ & $0,84^{\mathrm{c}}$ \\
\hline
\end{tabular}


Tableau 8 : Analyse de la variance du nombre de gousses, du poids sec des gousses, du nombre de graines et du poids sec des graines par plante au stade végétatif et au stade floraison.

\begin{tabular}{|c|c|c|c|c|c|c|c|c|c|}
\hline \multirow{3}{*}{ Source } & \multirow{3}{*}{ ddl } & \multicolumn{3}{|c|}{ Stade végétatif } & \multicolumn{5}{|c|}{ Stade floraison } \\
\hline & & ngse pt $^{-1}$ & pgse pt $^{-1}$ & ngrs pt ${ }^{-1}$ & psgrs pt $^{-1}$ & ngse pt $^{-1}$ & pgse pt $^{-1}$ & ngrs pt ${ }^{-1}$ & psgrs pt $^{-1}$ \\
\hline & & CM F & CM F & CM $\quad$ F & CM $\quad$ F & CM F & CM F & CM $\quad$ F & $\begin{array}{ll}\mathbf{C M} & \mathbf{F} \\
\end{array}$ \\
\hline $\mathrm{Rh}$ & 1 & $\begin{array}{l}82,57 * * \\
\mathrm{p}<0,0001\end{array}$ & $\begin{array}{l}21,97 * * \\
\mathrm{p}<0,0001\end{array}$ & $\begin{array}{l}928,28 * * \\
\mathrm{p}<0,0001\end{array}$ & $\begin{array}{l}9,42 * * \\
\mathrm{p}<0,0001\end{array}$ & $\begin{array}{l}185,78 * * \\
\mathrm{p}<0,0001\end{array}$ & $\begin{array}{l}56,59 * * \\
p<0,0001\end{array}$ & $\begin{array}{l}2162,57 * * \\
\mathrm{p}<0,0001\end{array}$ & $\begin{array}{l}38,56 * * \\
\mathrm{p}<0,0001\end{array}$ \\
\hline Compost & 6 & $\begin{array}{l}31,20 * * \\
\mathrm{p}<0,0001\end{array}$ & $\begin{array}{l}17,30 * * \\
\mathrm{p}<0,0001\end{array}$ & $\begin{array}{l}652,82 * * \\
\mathrm{p}<0,0001\end{array}$ & $\begin{array}{l}11,78 \\
* * \\
\mathrm{p}<0,0001\end{array}$ & $\begin{array}{l}17,37 * * \\
\mathrm{p}<0,0001\end{array}$ & $\begin{array}{l}11,40 * * \\
\mathrm{p}<0,0001\end{array}$ & $\begin{array}{l}470,01 * * \\
\mathrm{p}<0,0001\end{array}$ & $\begin{array}{l}9,50 * * \\
\mathrm{p}<0,0001\end{array}$ \\
\hline Rh*Compost & 6 & $\begin{array}{l}4,86 * * \\
\mathrm{p}=0,007\end{array}$ & $\begin{array}{l}2,8 * * \\
\mathrm{p}=0,004\end{array}$ & $\begin{array}{l}84,37 * * \\
\mathrm{p}<0,0001\end{array}$ & $\begin{array}{l}1,93 * * \\
\mathrm{p}=0,001\end{array}$ & $\begin{array}{l}9,87 \quad * * \\
\mathrm{p}<0,0001\end{array}$ & $\begin{array}{l}5,76 * * \\
\mathrm{p}<0,0001\end{array}$ & $\begin{array}{l}155,11 * * \\
\mathrm{p}<0,0001\end{array}$ & $\begin{array}{l}2,34 * * \\
p<0,0001\end{array}$ \\
\hline
\end{tabular}

par plante. Rh :régime hydrique. 


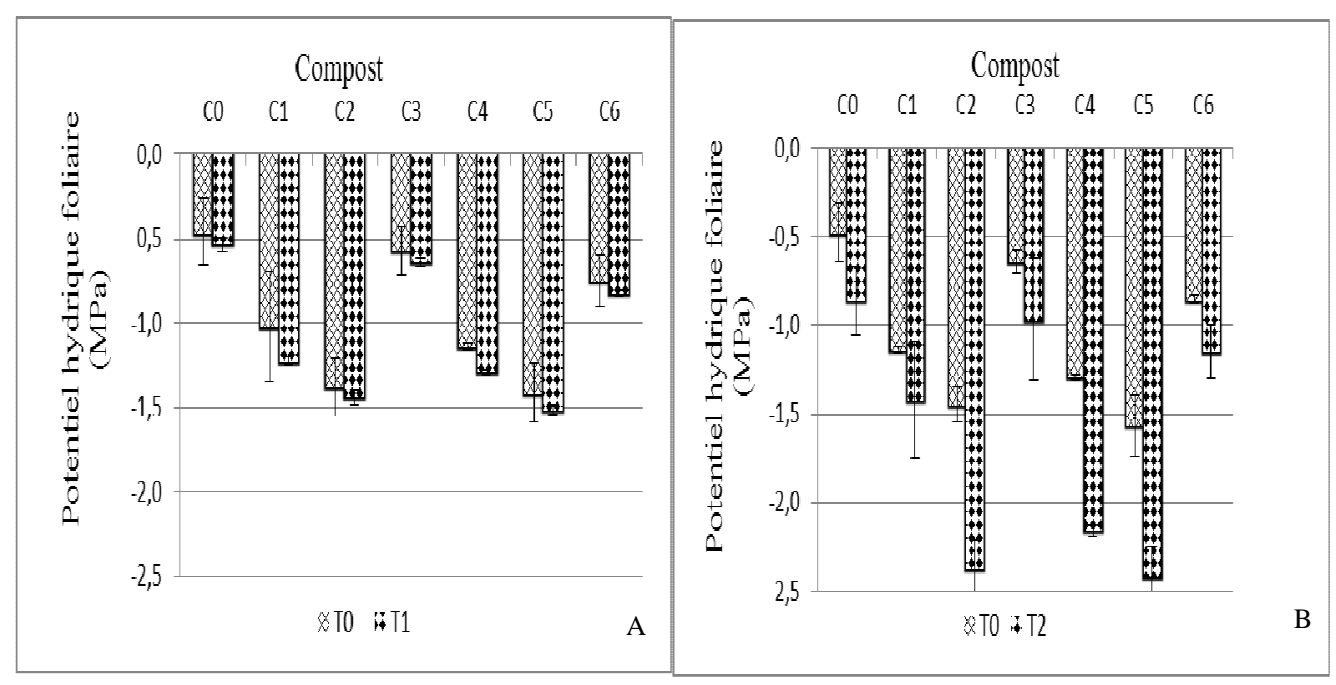

Figure 1: Potentiel hydrique foliaire en fonction du type de compost au 17è jais en phase végétative $(\mathrm{A})$ et au 14è jais en phase de floraison $(\mathrm{B})$. (T0= témoin avec une alimentation hydrique non limitant, $\mathrm{T} 1=$ suspension d'arrosage au stade végétatif ; $\mathrm{T} 2=$ suspension d'arrosage au stade floraison, jais $=$ jours après induction du stress.

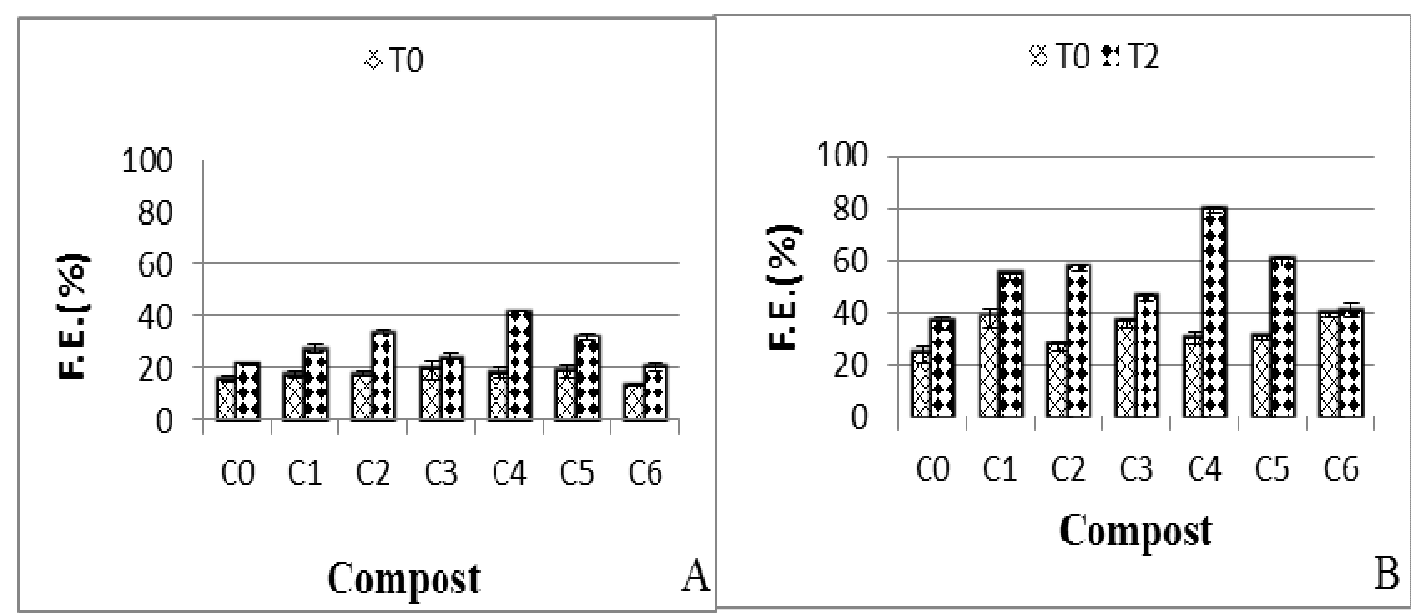

Figure 2 : Pourcentage de fuite d'électrolytes (FE) en fonction du type de compost au 17è jais en phase végétative $(\mathrm{A})$ et au $14 \mathrm{è} \mathrm{jais} \mathrm{en} \mathrm{phase} \mathrm{de} \mathrm{floraison}(\mathrm{B})$. ( $\mathrm{T} 0=$ témoin avec une alimentation hydrique non limitant, $\mathrm{T} 1=$ suspension d'arrosage au stade végétatif ; $\mathrm{T} 2=$ suspension d'arrosage au stade floraison). 


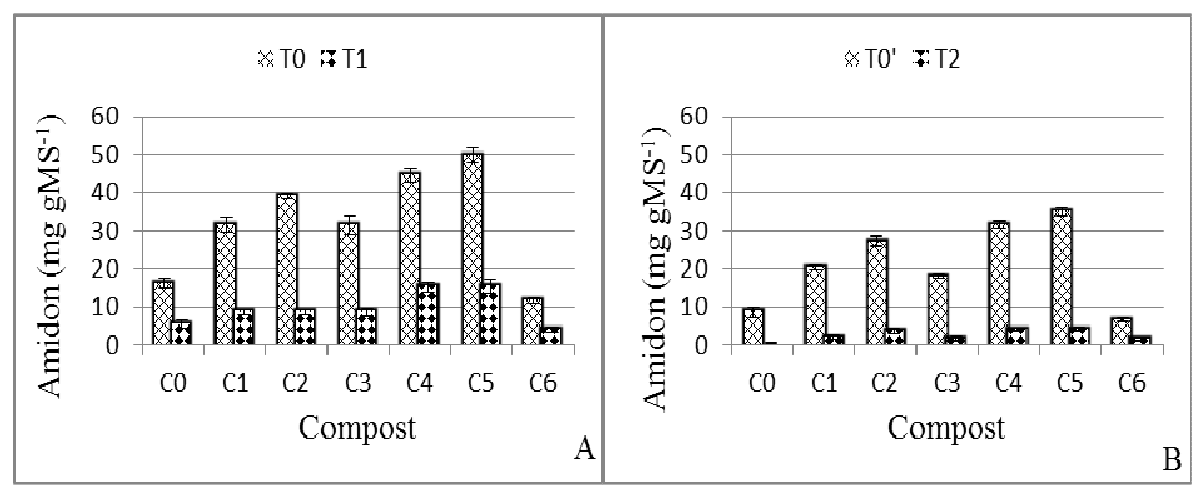

Figure 3: Teneur en amidon des feuilles en fonction du type de compost au 17è jais en phase végétative $(\mathrm{A})$ et au 14 è jais en phase de floraison $(\mathrm{B})$. (T0= témoin avec une alimentation hydrique non limitant, $\mathrm{T} 1=$ suspension d'arrosage au stade végétatif ; $\mathrm{T} 2=$ suspension d'arrosage au stade floraison).

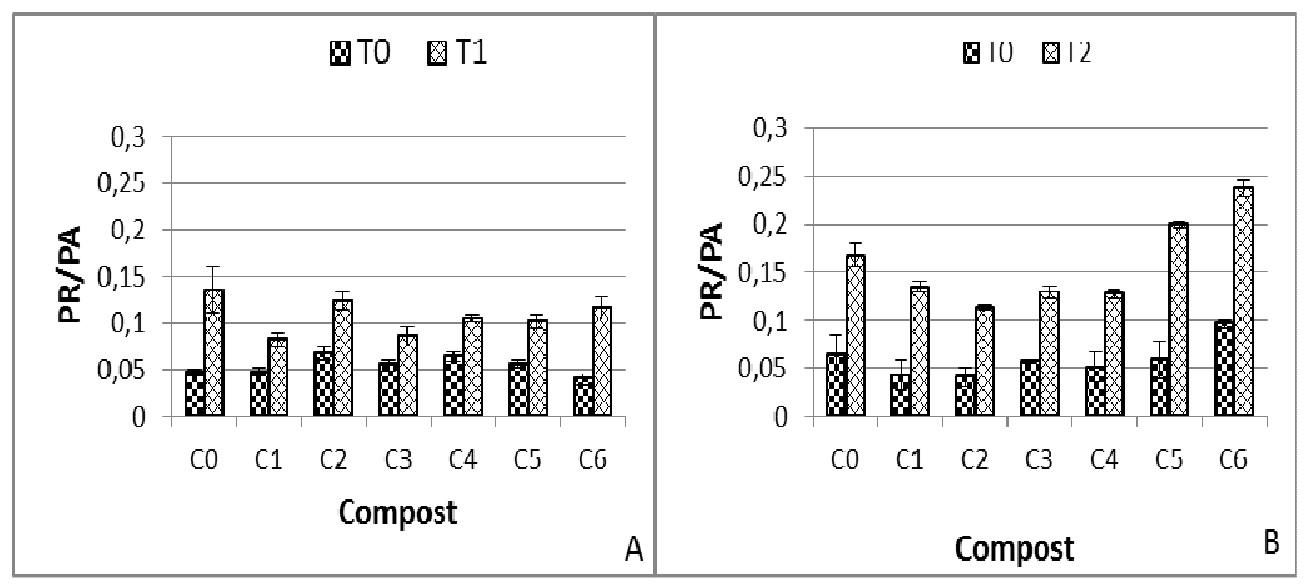

Figure 4 : Effet du stress hydrique sur le rapport PR/PA en fonction du type de compost au 17è jais en phase végétative $(\mathrm{A})$ et au 14è jais en phase de floraison $(\mathrm{B})$. (T0= témoin avec une alimentation hydrique non limitant, $\mathrm{T} 1=$ suspension d'arrosage au stade végétatif ; $\mathrm{T} 2=$ suspension d'arrosage au stade floraison).

\section{DISCUSSION}

Nos résultats montrent que les $\Psi$ fes plus faibles sont observées chez les plantes des composts $\mathrm{C} 1, \mathrm{C} 2, \mathrm{C} 4$ et $\mathrm{C} 5$ avec une moyenne de -1,4 MPa en T1 et -1,6 MPa en T2. Cela s'expliquerait par le fait que ces plantes possèdent plus de feuilles et un système racinaire plus développé. Ce qui induirait une transpiration importante et une forte absorption racinaire entraînant un assèchement accentué du sol. Ces deux paramètres physiologiques seraient réduits chez les plantes du sol témoin $\mathrm{C} 0$ et celles des composts $\mathrm{C} 3$ et $\mathrm{C} 6$ qui ont une biomasse arienne très réduite. Une grande surface foliaire et une croissance végétative rapide pourraient accélérer l'utilisation de l'eau et la diminution des réserves en eau du sol exposant les plantes à un déficit hydrique au milieu ou à la fin de leur cycle de développement (Passioura et Angus, 2010). Les composts $\mathrm{C} 1, \mathrm{C} 2, \mathrm{C} 4$ et $\mathrm{C} 5$ ont contribué à maintenir la croissance foliaire des plantes en augmentant le potentiel hydrique du sol. 
La hausse significative de fuite d'électrolytes observée chez les plantes stressées signifierait que le stress hydrique a affecté l'intégrité membranaire chez les plantes de tous les composts. Ces résultats confirment ceux obtenus par Falalou en 2006. L'absence de différence significative entre les pourcentages de fuites d'électrolytes des plantes témoins pourrait s'expliquer par le fait que nous avons une seule variété de niébé dont les protéines membranaires sont synthétisées sur la base des mêmes gènes. Les travaux de Diop (2002) ont mis en évidence des différences génotypiques sur l'intégrité membranaire du niébé en situation de stress. Par ailleurs, l'intégrité membranaire est beaucoup plus affectée au stade de floraison qu'au stade de végétatif. Ce qui montre que le déficit hydrique a été plus sévère au stade de floraison qu'au stade végétatif. Kirnak et al. (2001) ont lié l'augmentation des fuites d'électrolyte à la réduction des concentrations de chlorophylle (en raison de la sénescence des feuilles), tandis que McDonald et Archbold (1998) ont montré que la réduction de l'utilisation de l'eau affecte la perméabilité membranaire. La corrélation négative observée au stade floraison chez les stressées montre en effet que la diminution du Yf n'est pas suivie d'une diminution du pourcentage de fuite d'électrolytes mais au contraire son augmentation.

Chez les plantes non stressées un $\Psi \mathrm{f}$ élevé ne favorise pas une bonne accumulation de l'amidon dans les chloroplastes. Par contre une baisse importante du potentiel hydrique due à un déficit hydrique réduirait ou inhiberait la synthèse de l'amidon. Lors d'un déficit hydrique, l'activité physiologique de la feuille, et plus particulièrement la photosynthèse et la conductance stomatique sont affectées (Lowlor, 2002). La réduction de la photosynthèse, liée à la diminution du potentiel hydrique foliaire est supposée dépendre à la fois de la fermeture des stomates, avec pour conséquence une diminution de la conductance à la diffusion du $\mathrm{CO}_{2}$ et d'une limitation biochimique du chloroplaste à fixer le $\mathrm{CO}_{2}$ (Tardieu et Simoneau, 1998 ; Escolana et al., 1999;
Flexas et Medrano, 2002.). Nos résultats montrent que les stress $\mathrm{T} 1$ et T2 ont provoqué une diminution de la teneur en amidon chez les plantes pour tous les composts sauf pour le compost C6. L'hydrolyse de l'amidon en sucres solubles responsables l'osmorégulation, et/ou l'arrêt de sa synthèse due à l'inhibition de la photosynthèse par le stress hydrique pourrait(ent) expliquer cette baisse (Falalou, 2006). Pour le cas du compost C6 constitué uniquement de feuilles d'arbre, le maintien de la teneur des feuilles en amidon serait lié à sa grande capacité de rétention d'eau dans le sol, ce qui réduirait l'effet du stress sur le $\Psi$ f. La différence significative entre les composts $\mathrm{C} 1, \mathrm{C} 2, \mathrm{C} 3, \mathrm{C} 4, \mathrm{C} 5$ et le sol témoin $\mathrm{C} 0$ chez les plantes témoins pourrait s'expliquer par l'amélioration de la fertilité du sol due à la présence d'éléments minéraux fertilisants au sein de ces composts, à leur minéralisation progressive, à l'amélioration des caractéristiques physiques du sol.

Le déficit hydrique a induit une allocation de biomasse vers les racines, au détriment des parties aériennes au stade végétatif et au stade floraison pour les plantes de tous les traitements. Cette allocation de biomasse vers les racines s'est traduite aussi par des valeurs du rapport PR/PA toujours supérieures chez les plantes stressées (Albouchi et al., 2000). Le développement de la partie racinaire aux dépens de la partie aérienne est considéré par plusieurs auteurs comme un critère de résistance à la sécheresse Il permettrait une meilleure utilisation de l'eau disponible qui devient plus accessible (Thomas et Gausling, 2000).

La baisse des rapports PR/PA, observée chez les plantes stressées des composts $\mathrm{C} 1$, $\mathrm{C} 2$ et $\mathrm{C} 4$ au stade floraison peut s'expliquer par le fait que le système racinaire de ces plantes ayant bénéficié de l'allocation de biomasse durant le stade végétatif favoriserait une production de biomasse aérienne plus soutenue qu'au stade végétatif. Ces résultats concordent avec ceux rapportés par Werner et al. (2010), Gowda et al. (2012), et Vadez et al. (2012b) qui ont suggéré que l'augmentation de la croissance des racines en profondeur en 
condition de déficit hydrique améliore l'absorption de l'eau des plantes afin de maintenir un taux maximal de croissance aérienne. Cette réduction pourrait aussi résulter, en partie, de l'effet des restrictions imposées au système racinaire par le volume limité des pots. En effet, un enroulement des racines touchant les bords et particulièrement le fond des pots a été observé lors de la détermination des biomasses des différents plants. La différence d'évolution de la matière sèche entre le témoin et les traitements stressés n'était pas trop marquée au début du cycle végétatif du fait que la plante n'a pas encore développé un système foliaire vigoureux. En plein développement végétatif, cette différence apparaît nettement et l'écart maximal se situe au début de la formation des grains, période pendant laquelle la migration des assimilats des feuilles via les tiges vers les grains est importante.

D'un point de vue agronomique, la tolérance à la sécheresse est la capacité de la plante à croître et donner des rendements satisfaisants dans des zones sujettes à des déficits hydriques épisodiques (Chaves et Oliveira, 2002). Nos résultats montrent que les composantes du rendement ont été significativement réduites chez les plantes stressées par rapport aux plantes témoins sauf pour le nombre et le poids de gousses, le nombre de graines des plantes du compost C6. C6, à cause de sa grande capacité de rétention d'eau, arriverait à éviter la déshydratation et assurerait une production égale à celle du témoin. Par contre, c'est le compost qui a le moins assuré une bonne production par rapport aux autres. Ceci pourrait s'expliquer par sa minéralisation très lente.

\section{Conclusion}

L'objectif principal de l'étude a été de mesurer l'effet de six composts de déchets urbains solides (DUS) sur les quelques réponses morpho-physiologiques, biochimiques et agronomiques du niébé au stress hydrique. A l'issue de nos travaux, les résultats nous permettent de dire que tous les composts ont contribué à améliorer significativement la résistance au stress hydrique du niébé sauf le compost de déchets verts (C6) constitué uniquement de feuilles d'arbres.

Les amendements du sol par les composts dont les substrats initiaux comportent une proportion de déchets de cuisine (constitués de légumes) en l'occurrence $\mathrm{C} 2, \mathrm{C} 3, \mathrm{C} 4$ et $\mathrm{C} 5$ améliorent très significativement le développement foliaire et racinaire en condition témoin et en période de stress hydrique. Sur les plans physiologique et biochimique, les plantes de ces composts ont été affectées par le déficit hydrique au stade végétatif et de floraison. Nos résultats montrent des valeurs du potentiel hydrique foliaire très faibles et révèlent que l'intégrité membranaire a été affectée comparativement aux plantes du sol témoin et du compost des déchets d'abattoir (C1). Le stress hydrique a provoqué une réduction de la teneur en amidon des feuilles de ces plantes surtout au stade de floraison.

L'analyse des composantes du rendement en relation avec les paramètres physiologiques et biochimiques permet d'affirmer que les composts C2, C3, C4 et C5 augmentent le rendement en graines en condition de déficit hydrique et justifient l'intérêt du compostage des déchets de cuisine.

\section{REMERCIEMENTS}

Nous exprimons notre profonde gratitude au Pr. Edmond HIEN à l'Institut de Recherche pour le Développement (IRD) du Burkina pour son implication dans ce travail, pour l'encadrement et pour ses conseils. C'est lui qui nous a fourni les six types de composts et les échantillons du sol témoin prélevés dans son site d'expérimentation de Gampèla avec l'aide de son technicien M. Prosper SAWADOGO à qui nous adressons nos sincères remerciements.

\section{RÉFÉRENCES}

Agbicodo EM, Fatokun CA, Muranaka S, Visser RG, Linden van DCG. 2009. Breeding drought tolerant cowpea: constraints, accomplishments, and future prospects. Euphytica, 167: 353-370. 
Albouchi A, Sebeï H, Mezni MY, El Aouni MH. 2000, Influence de la durée d'une alimentation hydrique déficiente sur la production de biomasse, la surface transpirante et la densité stomatique d'Acacia cyanophylla Lindl. Annales de l'INRGREF, 4: 138-61.

Chaves MM, Oliveira MM. 2002. Mechanisms underlying plant resilience to water deficits: prospects for watersaving agriculture. Journal of Experimental Botany, 55: 2365-2384.

Collins NC, Tardieu F, Tuberosa R. 2008. Quantitative trait loci and crop performance under abiotic stress: Where do we stand? Plant Physiology, 147: 469486.

Diop NN. 2002. Ecophysiologie moléculaire de la tolérance à la sécheresse chez une Fabacée tropicale, Vigna unguiculata (L.) Walp., Clonage et étude d'un nouveau gène codant un inhibiteur de protéase. Thèse Doct., Univ. Paris XII, $138 \mathrm{p}$.

Escalona JM, Flexas J, Medrano H. 1999. Stomatal and non-stomatal limitations of photosynthesis under water stress in fieldgrown grapevines. Australian Journal of Plant Physiology, 26: 421-433.

Falalou H. 2006. Paramètres physiologiques, biochimiques et agronomiques pertinents pour les programmes d'amélioration et d'adaptation du niébé (Vigna unguiculata (L.) Walp.) au déficit hydrique. Thèse Doct., Université Ouagadougou, 169p.

FAO-ISRIC-ISSS (Food and Agriculture Organization of the United NationsInternational Soil Reference and Information Centre-International Society for Soil Science). 2006. World Reference Base for Soil Resources. FAO: Rome.

Fatokun C, Boukar O, Muranaka S, Chikoye D. 2009. Enhancing drought tolerance in cowpea. African Crop Science Society, 9: 531-536.

Flexas J, Medrano H. 2002. Droughtinhibition of photosynthesis in C3 plant: Stomatal and non-stomatal limitations revisited. Annals of Botany, 89: 183-189.
Gowda VR, Henry A, Vadez V, Shashidhar HE, Serraj R. 2012. Water uptake dynamics under progressive drought stress in diverse accessions of OryzaSNP panel of rice (Oryza sativa L.). Functional Plant Biology, doi: 10.1071/FP12015

Hall AE, Cisse N, Thiaw S, Elawad HOA, Ehlers JD, Ismail AM, Fery R, Roberts PA, Kitch LW, Murdock LL, Boukar O, Phillips RD, McWatters KH. 2003. Development of cowpea cultivars and germplasm by the Bean / Cowpea CRSP. Field Crops Research, 82: 103-134.

Hien E. 2004. Dynamique du carbone dans un acrisol ferrique du Centre Ouest Burkina: Influence des pratiques culturales sur le stock et la qualité de la matière organique, $\mathrm{PhD}$ Thesis Ecole Nationale Supérieure Agronomique de Montpellier, France, 140p.

IPCC. 2007. Climate change: the physical science basis. Contribution of working group I to the fourth assessment report of the intergovernmental panel on climate change (IPCC). Cambridge University Press, Cambridge. p. 996

Jarvis CE, Walker J. 1993. Simultaneous, rapid spectrophotometric determination of total starch amylose and amylopectin. J. Sci. Food Agric., 63 : 53-57.

Kabore WT. 2004. Amélioration de la Valorisation Agricole des Déchets Urbains après Compostage : Influence de la Nature et des Proportions des Substrats Initiaux sur les Valeurs Amendante et Fertilisante des Composts. Thèse Doct., Université Ouagadougou, 248p.

Kirnak H, Cengiz K, David H, Sinan G. 2001. A long-term experiment to study the role of mulches in physiology and macronutrition of strawberry grown under water stress. Austral. J. Agricult. Res., 52(9): 937-943.

Ksontini M. 1996. Etude écophysiologique des réponses à la contrainte hydrique du chêne liège (Quercus suber) dans le Nord-Tunisie : comparaison avec le chêne Kermès $(Q$. coccifera) et le chêne zen $(Q$. 
faginea). Thèse Doct, univ Paris XII-Val de Marne, $157 \mathrm{p}$.

Lowlor DW. 2002. Limitation to photosynthesis to water stressed leaves: stomata vs. metabolism and the role of ATP. Annals of Botany, 89: 871-885

McDonald S, Archbold D. 1998. Membrane competence among and within Fragaria species varies in response to dehydration stress. J. Am. Soc. Hortic. Sci., 123(5) : 808-813.

Morel JL. 1982. L'évaluation de la maturité des composts urbains par une métode colorimétrique. Compost-information, 10:4-8.of superoxide dismutase and catalase. J. Exp. Bot., 32: 93-101.

Muchero W, Ehlers JD, Roberts PA. 2008. Seedling stage drought-induced phenotypes and drought-responsive genes in diverse cowpea genotypes. Crop Science, 48: 541-552.

Ogbonnaya CI, Sarr B, Brou C, Diouf O, Diop NN, Roy-Macauley H. 2003. Selection of cowpea genotypes in hydroponics, pots, and field for drought tolerance. Crop Science, 43: 1114-1120.

Passioura JB, Angus JF. 2010. Improving productivity of crops in water-limited environments. In Advances in Agronomy, Sparks DL (ed). Academic Press: Burlington; 37-75.

Salack S, Muller B, Gaye AT. 2011. Rainbased factors of high agricultural impacts over Senegal. Part I: integration of local to sub-regional trends and variability. Theorical \& Applied Climatology, 106 (1-2): 1-22.

Singh BB, Ajeigbe HA, Tarawali SA, Fernandez-Rivera S, Abubakar M. 2003. Improving the production and utilization of cowpea as food and fodder. Field Crops Research, 84: 169-177.

Singh BB, Awika J. 2010. Breeding diverse, durable and diet-plus cowpea varieties for increased production, enhanced nutrition and health in the 21st century. In Book of abstracts of the 5th World Cowpea
Conference, Fatokun CA (ed). Saly: Senegal; 16.

Thiaw S. 2003. Association between slow leaf electrolyte leakage under heat stress and heat tolerance during reproductive development in cowpea. Ph.D., California Riverside University, 100p.

Thomas FM, Gausling T. 2000, Morphological and physiological responses of oak seedlings (Quercus petraea and Q. robur) to moderate drought. Ann For Sci., 57: 325-33.

Tradieu F, Simoneau. 1998. Variability among species of stomatal control under fluctuating soil water status and evaporative demand: modeling isohydric and anisohydric behaviours. Journal of Experimental Botany, 49: 419-432.

Tripathy JN, Zhang J, Robin S, Nguyen ThT, Nguyen HT. 2000. QTLs for cellmembrane stability mapped in rice (Oriza sativa L.) under drought stress. Theor. Appl. Genet., 100: 1197-1202.

Vadez V, Rao S, Bhatnagar Mathur P, Sharma KK. 2012. DREB1A promotes root development in deep soil layers and increases water extraction under water stress in groundnut. Plant Biology, doi: 10.1111. /j.1438-8677.2012.00588.x.

Werner T, Nehnevajova E, Kollmer I, Novak O, Stmad M, Kramer U, Schmulling T. 2010. Root-specific reduction of cytokinin causes enhanced root growth, drought tolerance, and leaf mineral enrichment in Arabidopsis and tobacco. Plant Cell, 22: 3905-3920.

Wittig R, Konig K, Schmidt M, Szarzynski J, 2007. A study of climate change and anthropogenic impacts in West Africa. Environmental Science \& Pollution Research, 14: 182-189.

Yang S, Vanderbeld B, Wan J, Huang Y, 2010. Narrowing down the targets: towards successful genetic engineering of drought-tolerant crops. Molecular Plant 3: $469-490$. 